\title{
Biomarkers for renal transplantation: where are we?
}

This article was published in the following Dove Press journal: International Journal of Nephrology and Renovascular Disease 30 September 2013 Number of times this article has been viewed

\section{Fangmin $\mathrm{Ge}^{1-3}$ Qiaoding Dai ${ }^{4}$ Weihua Gong 5}

'Hospital Administration Office, ${ }^{2}$ Department of Medicine, Second Affiliated Hospital Zhejiang University School of Medicine, ${ }^{3}$ Health Bureau of Shangcheng District Hangzhou, ${ }^{4}$ Department of Rheumatism and Immunology, First Affiliated Hospital of Zhejiang Chinese Medicine University, ${ }^{5}$ Department of Surgery, Transplant International Research Centre, Second Affiliated Hospital Zhejiang University School of Medicine, Hangzhou, People's Republic of China
Correspondence: Weihua Gong Department of Surgery, Transplant International Research Centre, Second Affiliated Hospital Zhejiang University School of Medicine, Jiefang Road \# 88, Hangzhou, Zhejiang Province 310009 , People's Republic of China

Tel +86 57I 87783580

Fax +86 57। 8778358 I

Email weihua.gong@gmail.com
Abstract: Although surgical techniques, post-transplant care medicine, and immunosuppressants have been greatly improved, permanent acceptance of renal allograft remains a clinical challenge owing to the appearance of various influencing factors. To predict graft dysfunction, development of noninvasive biomarkers is becoming a highlighted research topic in the field of renal transplantation, which provides a possibility for physicians to give preemptive rescue treatment. From the viewpoint of diagnostic techniques, repetitive sampling is prerequisite to identify applicable biomarkers in the clinic. Early biomarkers can be used to dynamically monitor renal graft status and accurately predict transplant outcome independent of various confounders. This review highlights recent studies on the predictive value of biomarkers and methods to quantify biomarkers for monitoring kidney transplant. It is important to analyze and compare different biomarkers for living, and nonliving donors. Analysis of identified clinically relevant biomarkers will advance our understanding of distinct molecular and cellular mechanisms of transplantation and provide insight into developing novel potential approaches to induce transplant tolerance.

Keywords: biomarkers, renal transplantation, diagnostic techniques, reversible, preemptive treatment

\section{Introduction}

Currently, kidney transplantation remains an efficient and major therapeutic strategy for end-stage renal disease. Although surgical techniques and postoperative care have greatly advanced, achievement of long-term allograft survival remains a clinical challenge owing to various confounders, such as viral infection and nephrotoxicity. ${ }^{1}$ Therefore, earlier detection and identification of renal graft status may guide clinicians in minimizing the risk of graft rejection events and provide decisive advice regarding withdrawal of immunosuppression. ${ }^{1}$ Subsequent preemptive treatment can be adopted to prevent irreversible damage of graft and rescue allograft (Figure 1). To this end, efficient implementation of biomarker identification can realize personalized therapy for renal transplant patients. ${ }^{2}$

Indeed, biomarkers for monitoring graft function have been studied for decades. ${ }^{1,3}$ As molecular biological techniques are developing, various types of biomarkers have been successfully identified (Table 1 ); ${ }^{1}$ however, no suitable biomarkers are widely used for renal transplant patients since clinical cases are normally complicated. Much of the data from experimental findings failed to connect bedside clinical applications. Therefore, standardization of identified biomarkers is required on a large scale in prospective multicenter trials. $^{2}$ 


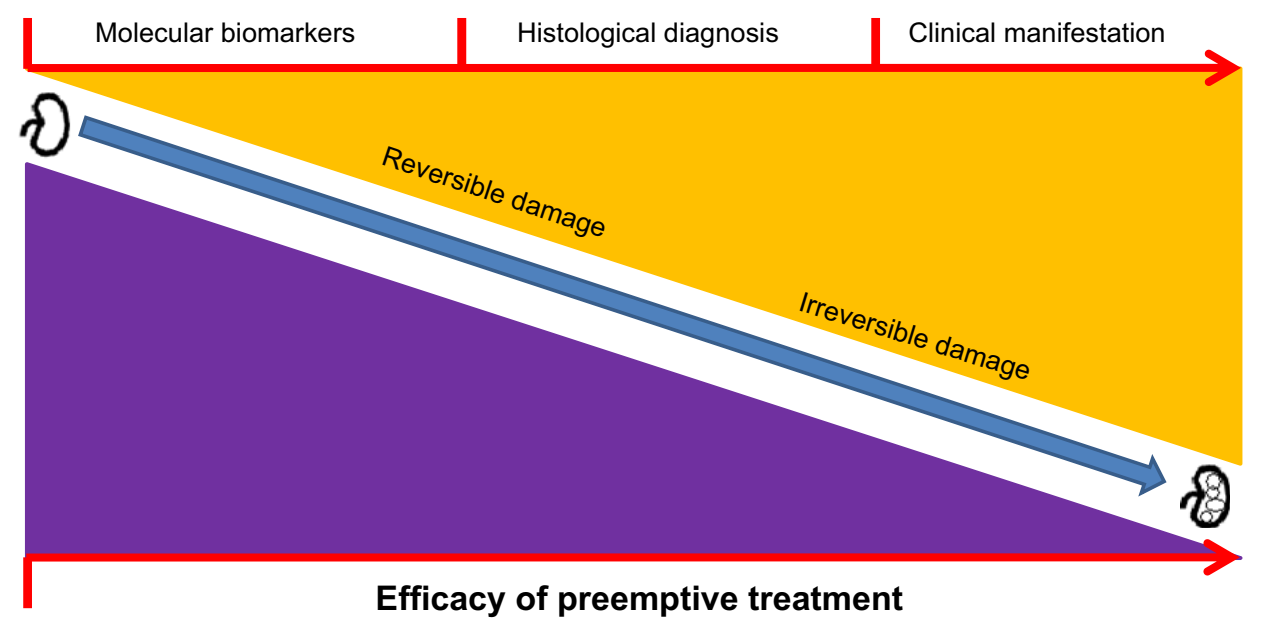

Figure I Schematic illustration of progress of renal graft damage, diagnostic techniques, and efficacy of preemptive treatment.

Notes: After kidney transplantation, the allograft undergoes various injurious factors, such as ischemia-reperfusion, brain death, cardiac death, etc. Diagnostic techniques are applied to identify the progress of graft damage by using molecular biomarkers, histological diagnosis, and clinical analysis. The early utilization of suitable biomarkers might create a window for reversing tissue damage, which will improve efficacy of preemptive treatment.

In practice, the ideal biomarkers for renal transplantation should be independently validated and standardized by multiple international centers, ${ }^{4,5}$ which can prove their reliability and reproducibility in parallel with corresponding histological analysis. It is encouraging that the European Union Reprogramming the Immune System for the Establishment of Tolerance Consortium undertook such collaborative studies in different European laboratories. ${ }^{2}$ Bestard et al presented their very recent data in which a cross-validation of the interferon gamma enzyme-linked immunosorbent spot assay was used to assess the circulating alloreactive memory/effector T-cells for renal transplant patients. ${ }^{5}$ Similarly, 12 US pediatric transplant programs cross-validated and analyzed a five gene-set (NKTR, PBEF1, MAPK9, DUSP1, PSEN1) with high accuracy for acute rejection in clinical renal transplantation. ${ }^{4}$ Ideal biomarkers should also be capable of sensitively and specifically monitoring allograft function, independent of various confounding factors such as the influence of cytomegalovirus infection or donors from brain death (DBD). ${ }^{1}$ Early identification and prognosis of graft dysfunction are also required so that a preemptive regimen can be promptly administered to prevent irreversible damage to the allograft. In addition, optimal biomarkers are expected to precisely reflect alteration of allograft function at an early stage. ${ }^{1}$

Herein, our present review analyzes laboratory techniques for identifying biomarkers to better understand the development of diagnostic tools. Current studies on biomarkers for living and nonliving donors are discussed to acknowledge their predictive values and practical considerations.

\section{Development of laboratory techniques for identifying novel biomarkers}

Since creatinine cannot be used as an early marker for predicting or monitoring renal graft function, great efforts have been made to explore suitable biomarkers or parameters to prevent

Table I Biomarkers for specific conditions of donor kidney grafts

\begin{tabular}{|c|c|c|c|c|c|}
\hline Donors & $\begin{array}{l}\text { Various } \\
\text { scenarios }\end{array}$ & Laboratory techniques & $\begin{array}{l}\text { Biomarker } \\
\text { source }\end{array}$ & $\begin{array}{l}\text { Potential } \\
\text { biomarkers }\end{array}$ & References \\
\hline \multirow{5}{*}{$\begin{array}{l}\text { Living } \\
\text { donors }\end{array}$} & \multirow[t]{5}{*}{ Living donors } & Luminex and/or single antigen beads & Peripheral blood & DSA & 13 \\
\hline & & ELISA & Perfusate & NGAL & 15 \\
\hline & & ELISA & Urine & NGAL & 14 \\
\hline & & TaqMan microRNA assays & Renal allograft biopsy & microRNAs:miR-I42-5p, & 18,19 \\
\hline & & RT-PCR & Peripheral blood & miR-I55, miR-223 & \\
\hline Nonliving & Deceased donors & Microarray profiling & Renal graft & CXCR4, CCL5, ITGB2 & 8 \\
\hline \multirow[t]{2}{*}{ donors } & $\mathrm{DCD}$ & ELISA & Perfusate & LDH, IL-I8 & 7 \\
\hline & DBD & Mass spectrometry analysis & Serum & ACY-I & 9 \\
\hline
\end{tabular}

Abbreviations: ACY-I, aminoacylase-I; CCL5, chemokine (C-C motif) ligand 5; CXCR4, CXC-chemokine receptor 4; DBD, donor from brain death; DCD, donor from cardiac death; DSA, donor-specific antibodies; ELISA, enzyme-linked immunosorbent assay; IL-I8, interleukin-I8; ITGB2, integrin beta 2; LDH, lactate dehydrogenase; NGAL, neutrophil gelatinase-associated lipocalin; RT-PCR, real-time polymerase chain reaction; microRNA, micro-ribonucleic acid. 
the occurrence of graft impairment. ${ }^{1,6}$ Indeed, the development of laboratory techniques has facilitated the production of novel biomarkers. Firstly, the real-time polymerase chain reaction (RT-PCR) is a sensitive and accurate technique, wellaccepted to identify and validate potential biomarkers at the gene level for decades. ${ }^{1}$ However, the data from RT-PCR is insufficient for high throughput screening and cannot meet researchers' needs. As a replacement, microarray profiling arose to generate a large amount of potential biomarkers for comprehensive analysis on various renal transplant patients in different transplant scenarios ${ }^{4,7,8}$ During the past years, mass spectrometry analysis was employed to identify potential biomarkers at the protein level in patients' serum samples. ${ }^{9}$ Apart from specimens from peripheral blood, urine was utilized to identify qualified biomarkers for early detection of glomerular injury. ${ }^{6}$ Research findings exhibited that urinary cystatin $\mathrm{C}$, clusterin, and beta- 2 microglobulin could significantly monitor kidney injury better than serum creatinine or blood urea nitrogen, as supported by in situ hybridization, gene and protein expression analysis, and immunohistochemistry. Other multiple platforms are also used in empirical studies, such as enzyme-linked immunosorbent assay, flow cytometry, and immune cell functional assays, although their efficiency is unsatisfactory (Table 1). ${ }^{10,11}$ In addition, perfusate was utilized to detect applicable noninvasive biomarkers for renal transplant patients (Table 1 ).

\section{Different types of biomarkers for renal allograft monitoring in kidney transplantation}

Diverse confounding factors such as viral infection, malignancy, surgical complication, and nephrotoxicity from immunosuppressive drugs can affect graft function, and even survival. ${ }^{1}$ Therefore, specific biomarkers were investigated for such varying clinical scenarios of kidney transplantation. ${ }^{7-9}$

First, transplant organs can be categorized into those received from living donors, and those from deceased donors. To some extent, the quality of donor organs determines transplant outcome. ${ }^{12}$ It is well known that living donor organs function better than those from deceased donors. Accordingly, the biomarkers for kidney transplantation can be divided into two types: biomarkers for living donors, and biomarkers for deceased donors. ${ }^{8}$

\section{Biomarkers for living donor kidneys}

Although living donors benefit patients in regard to long-term graft survival, no protocols for perfect tolerance induction exist in empirical transplantation. Graft dysfunction is always observed from a living donor. A study on 72 living-donor kidney transplant recipients who received total lymphoid irradiation or bortezomib was performed to identify sensitive biomarkers for preventing graft failure. ${ }^{13}$ The findings exhibited that the level of donor-specific antibodies (DSA) steadily increased post-transplantation. Administration of prednisone could reduce DSA production, and an inverse association between prednisone dose and peripheral DSA level was observed (risk ratio $=0.92$ ). The study indicated that monitoring DSA level might predict alteration of immune reactivity and may help determine the weaning process from immunosuppressive drugs. ${ }^{13}$ Another interesting study revealed that the level of neutrophil gelatinase-associated lipocalin (NGAL) in the urine might be a sensitive biomarker reflecting acute renal allograft dysfunction. This biomarker was validated by immunohistochemical analysis. ${ }^{14}$ NGAL is mainly released by activated neutrophils and can accumulate in the proximal tubule after acute tubular injury. ${ }^{15}$ As an acute rejection event occurred, NGAL protein expression was significantly increased in the proximal tubules of the living-donor kidney graft. Furthermore, it was observed that the peripheral NGAL level was sensitively augmented. ${ }^{14}$ Data from porcine renal transplantation support these findings, in that NGAL concentration in perfusate could be utilized to reflect machine-perfused kidney injury and indicated renal graft function after transplantation. ${ }^{15}$

Since the first micro-ribonucleic acid (microRNA), a small noncoding molecule, was discovered in 1993, ${ }^{16}$ microRNAs have been widely studied, particularly in the regulation of immune cells including innate and adaptive immune responses. ${ }^{17}$ An important study by Anglicheau et al on the role of microRNA biomarkers in transplantation exhibited that high expression of microRNA (miR)-142-5p, miR-155, and miR-223 in the peripheral blood mononuclear cells and renal allograft biopsy of living donor transplant recipients were predictive of an acute rejection event. ${ }^{18}$ Danger et al validated the biomarker miR-142-5p in the peripheral blood mononuclear cells, which can reflect chronic antibody-mediated rejection of renal transplant patients. ${ }^{19}$

\section{Biomarkers for nonliving donor kidneys}

Outcome of nonliving donor transplants is worse than that of living donor transplants. ${ }^{12}$ Nonliving donor kidneys can cause various postoperative complications, such as delayed graft function (DGF). ${ }^{20}$ Early graft function is a crucial determinant of long-term kidney transplant outcome; ${ }^{21}$ therefore, 
it is of great interest to summarize the various biomarkers after nonliving donor kidney transplantation.

DGF and primary nonfunction (PNF) frequently occur in the first week of graft life, which has a deleterious effect on long-term allograft survival. ${ }^{7,22}$ They may be caused by multiple factors, such as utilization of $\mathrm{DBD},{ }^{22}$ donors from cardiac death (DCD), ${ }^{7}$ or there may be a weight difference between the donor and recipient, resulting in acute tubular necrosis, post-transplantation oliguria, and even an increased risk of acute rejection episodes. ${ }^{23}$ Indeed, post-transplantation renal allograft function of DCD recipients is comparable to that of DBD patients, although their influential mechanisms are different. ${ }^{22,24}$ Therefore, early identification of DGF and PNF is of great value and interest for clinicians and researchers, which will benefit prognostic stratification of renal transplant patients and eventually improve transplant outcome. ${ }^{9}$ An intriguing question is whether the same biomarkers can be identified and used to monitor allograft function.

Brain death remains a clinical challenge to be overcome in the field of transplantation, causing hormonal alteration, neuroimmunologic effects, and hemodynamic instability. Consequently, a cascade of inflammatory events is unleashed, leading to exacerbation of ischemia/reperfusion injury and an impaired graft survival. ${ }^{25}$ To identify biomarkers for DBD transplant recipients, a study was performed by Welberry Smith et al. ${ }^{9}$ Their findings revealed that serum aminoacylase-1 (ACY-1) levels at day 1 or 3 post-transplant were significantly associated with delayed, slow, and immediate graft function, particularly dialysis-free survival, by using proteomic analysis of long-term follow-up of 54 renal transplant patients. ${ }^{9}$ Furthermore, an independent confirmative cohort study was employed among 194 patients to validate the association between serum ACY-1 level and incidence of DGF. ${ }^{9}$ Indeed, ACY-1 can be expressed in the pan-tubule and predominantly proximal tubule of pig and human kidneys. ${ }^{9}$ It is reasonable to explain that DGF causes tubular damage and a significant increase of serum ACY-1 level. ${ }^{9}$

Donor from cardiac death can also result in DGF and even PNF. A very recent attempt was made to identify accurate early biomarkers from perfusate to predict the occurrence of DGF and PNF. ${ }^{7}$ The establishment of a biomarker prior to implantation would provide a possibility to prevent the process of graft injury. It was found that lactate dehydrogenase and interleukin-18 concentrations in the preservation solution of machine-perfused kidneys were associated with PNF and DGF, but there was no significant association with 1-year graft survival. ${ }^{7}$ Therefore, more studies are required to investigate biomarkers for DCD-related graft dysfunction.
With respect to a single factor of deceased-donor graft, three critical genes (CXCR4, CCL5, and ITGB2) were identified from 112 specimens by using microarray profiling examination. Based on a random forest analysis, these were suggested to be suitable biomarkers to assess organ quality and predict kidney graft function. ${ }^{8}$ Nevertheless, further prospective studies are absent.

\section{Combined use of biomarkers}

Indeed, implementation of more biomarkers can significantly improve predictive value. Based on our own studies, Foxp3 gene expression in the peripheral blood might be dramatically increased at early stage post-transplantation, after an acute rejection episode has occurred. However, this increased level was insufficiently differentiated from that of chronically rejecting recipients, which hampered its application as a suitable biomarker. Interestingly, the ratio of Foxp3/alpha1,2-manosidase might monitor allograft function and predict long-term transplant outcome ${ }^{26,27}$ but alpha-1,2-manosidase alone does not (Weihua Gong, unpublished data, 2008). Therefore, combined use of biomarkers is necessary and will enable identification of patients suitable for preemptive treatment, particularly for those at risk of rejection, overdose of immunosuppression, and nephrotoxicity.

\section{Future directions}

As experimental transplant models are improving, a considerable number of potential biomarkers are being identified, ${ }^{15,28}$ including more biomarkers for living or nonliving donors. Suitable biomarkers available for both patient populations are required. It is well-accepted that the great advances in translational medicine can potentiate validation of these identified biomarkers from bench to bed in a transplant center. Nevertheless, international standardization of biomarkers is still required to test their predictive power through prospective multicenter studies, ${ }^{29,30}$ and their reliability and reproducibility will be accordingly validated. ${ }^{2}$

The sensitivity and specificity of these biomarkers for monitoring allograft function is an important issue, particularly in the settings of various confounders. ${ }^{1}$ Combined use of biomarker patterns might provide reliable and significant indication on allograft function, which might shed light on the appropriate preemptive therapeutic strategies. ${ }^{1}$ Furthermore, systematic analysis using bioinformatics tools can be utilized to avoid repetitive scientific research. Taken together, our goal is to achieve long-term allograft survival using personalized biomarkers. 


\section{Acknowledgments}

This work was supported by the Department of Education of Zhejiang Province (Grant No Y201226017), the National Natural Science Foundation of China (No 81270323), the Qianjiang Talents Project of Zhejiang Province (Grant No 2012R10022), the Young Eagles Program of the First Affiliated Hospital of Zhejiang Chinese Medicine University, and Zhejiang Provincial Outstanding Youth Foundation (Grant No LR13H020001).

\section{Disclosure}

The authors report no conflicts of interest in this work.

\section{References}

1. Gong W, Whitcher GH, Townamchai N, Xiao X, Ge F. Biomarkers for monitoring therapeutic side effects or various supratherapeutic confounders after kidney transplantation. Transplant Proc. 2012;44(5):1265-1269.

2. Sawitzki B, Pascher A, Babel N, Reinke P, Volk HD. Can we use biomarkers and functional assays to implement personalized therapies in transplantation? Transplantation. 2009;87(11):1595-1601.

3. Pantschewa-Haschen R, Schulze R, Schneider G, Schabel J. [Urinary enzymes in monitoring kidney transplant patients.] Z Urol Nephrol. 1980;73(10):719-724. German [with English abstract].

4. Li L, Khatri P, Sigdel TK, et al. A peripheral blood diagnostic test for acute rejection in renal transplantation. Am J Transplant. 2012;12(10):2710-2718.

5. Bestard O, Crespo E, Stein M, et al. Cross-validation of IFN- $\gamma$ Elispot assay for measuring alloreactive memory/effector $\mathrm{T}$ cell responses in renal transplant recipients. Am J Transplant. 2013;13(7):1880-1890.

6. Dieterle F, Perentes E, Cordier A, et al. Urinary clusterin, cystatin C, beta2-microglobulin and total protein as markers to detect drug-induced kidney injury. Nat Biotechnol. 2010;28(5):463-469.

7. Hoogland ER, de Vries EE, Christiaans MH, Winkens B, Snoeijs MG, van Heurn LW. The value of machine perfusion biomarker concentration in DCD kidney transplantations. Transplantation. 2013;95(4):603-610

8. Scian MJ, Maluf DG, Archer KJ, et al. Identification of biomarkers to assess organ quality and predict posttransplantation outcomes Transplantation. 2012;94(8):851-858.

9. Welberry Smith MP, Zougman A, Cairns DA, et al. Serum aminoacylase-1 is a novel biomarker with potential prognostic utility for long-term outcome in patients with delayed graft function following renal transplantation. Kidney Int. Epub 2013 Jun 5.

10. Wen R, Wu V, Dmitrienko S, Yu A, Balshaw R, Keown PA; Genome Canada Biomarkers in Transplantation Group. Biomarkers in transplantation: prospective, blinded measurement of predictive value for the flow cytometry crossmatch after negative antiglobulin crossmatch in kidney transplantation. Kidney Int. 2006;70(8):1474-1481.

11. Vyas S, Roberti I. Lymphocyte ATP immune cell function assay in pediatric renal transplants: is it useful? Transplantation Proc. 2011;43(10): 3675-3678.
12. Matas AJ, Payne WD, Sutherland DE, et al. 2,500 living donor kidney transplants: a single-center experience. Ann Surg. 2001;234(2): 149-164.

13. Hoshino J, Kaneku H, Everly MJ, Greenland S, Terasaki PI. Using donor-specific antibodies to monitor the need for immunosuppression. Transplantation. 2012;93(11):1173-1178.

14. Kohei J, Ishida H, Kazunari T, Tsuchiya K, Nitta K. Neutrophil gelatinase-associated lipocalin is a sensitive biomarker for the early diagnosis of acute rejection after living-donor kidney transplantation. Int Urol Nephrol. 2013;45(4):1159-1167.

15. Jochmans I, Monbaliu D, Pirenne J. Neutrophil gelatinase-associated lipocalin, a new biomarker candidate in perfusate of machine-perfused kidneys: a porcine pilot experiment. Transplant Proc. 2011;43(9):3486-3489.

16. Lee RC, Feinbaum RL, Ambros V. The C elegans heterochronic gene lin-4 encodes small RNAs with antisense complementarity to lin-14. Cell. 1993;75(5):843-854.

17. Lodish HF, Zhou B, Liu G, Chen CZ. Micromanagement of the immune system by microRNAs. Nat Rev Immunol. 2008;8(2):120-130.

18. Anglicheau D, Sharma VK, Ding R, et al. MicroRNA expression profiles predictive of human renal allograft status. Proc Natl Acad Sci U S A. 2009;106(13):5330-5335.

19. Danger R, Paul C, Giral M, et al. Expression of miR-142-5p in peripheral blood mononuclear cells from renal transplant patients with chronic antibody-mediated rejection. PloS One. 2013;8(4):e60702.

20. Premasathian N, Avihingsanon Y, Ingsathit A, Pongskul C, Jittiganont S, Sumethkul V. Risk factors and outcome of delayed graft function after cadaveric kidney transplantation: a report from the Thai Transplant Registry. Transplant Proc. 2010;42(10):4017-4020.

21. Parekh J, Bostrom A, Feng S. Diabetes mellitus: a risk factor for delayed graft function after deceased donor kidney transplantation. Am J Transplant. 2010;10(2):298-303.

22. Siedlecki A, Irish W, Brennan DC. Delayed graft function in the kidney transplant. Am J Transplant. 2011;11(11):2279-2296.

23. Gong W, Klöpfel M, Reutzel-Selke A, et al. High weight differences between donor and recipient affect early kidney graft function - a role for enhanced IL-6 signaling. Am J Transplant. 2009;9(8):1742-1751.

24. Wadei HM, Heckman MG, Rawal B, et al. Comparison of kidney function between donation after cardiac death and donation after brain death kidney transplantation. Transplantation. 2013;96(3):274-281.

25. Floerchinger B, Oberhuber R, Tullius SG. Effects of brain death on organ quality and transplant outcome. Transplant Rev (Orlando). 2012;26(2):54-59.

26. Sawitzki B, Reinke P, Volk HD, Wood K, Turka LA. Autoimmunity and transplantation: a meeting at the crossroads in Berlin. Nat Immunol. 2008;9(5):447-449.

27. Sawitzki B, Bushell A, Steger U, et al. Identification of gene markers for the prediction of allograft rejection or permanent acceptance. Am J Transplant. 2007;7(5):1091-1102.

28. Thuillier R, Codas R, Marchand E, et al. Chronic renoprotective effect of pulsatile perfusion machine RM3 and IGL-1 solution in a preclinical kidney transplantation model. J Transl Med. 2012;10:233.

29. Brouard S, Ashton-Chess J, Soulillou JP. Surrogate markers for the prediction of long-term outcome in transplantation: Nantes Actualité Transplantation (NAT) 2007 meeting report. Hum Immunol. 2008;69(1):2-8.

30. Parikh CR, Edelstein CL, Devarajan P, Cantley L. Biomarkers of acute kidney injury: early diagnosis, pathogenesis, and recovery. J Investig Med. 2007;55(7):333-340.

International Journal of Nephrology and Renovascular Disease

\section{Publish your work in this journal}

The International Journal of Nephrology and Renovascular Disease is an international, peer-reviewed open-access journal focusing on the pathophysiology of the kidney and vascular supply. Epidemiology, screening, diagnosis, and treatment interventions are covered as well as basic science, biochemical and immunological studies. The journal welcomes

\section{Dovepress}

original research, clinical studies, reviews \& evaluations, expert opinion and commentary, case reports and extended reports. The manuscript management system is completely online and includes a very quick and fair peerreview system, which is all easy to use. Visit http://www.dovepress.com/ testimonials.php to read real quotes from published authors 